\title{
Science, misinformation and digital technology during the Covid-19 pandemic
}

\author{
Aníbal Monasterio Astobiza ${ }^{1,2}$ (D)
}

Received: 31 August 2020 / Accepted: 26 April 2021 / Published online: 11 May 2021

(C) Springer Nature Switzerland AG 2021

\begin{abstract}
Three interdependent factors are behind the current Covid-19 pandemic distorted narrative: (1) science's culture of "publish or perish", (2) misinformation spread by traditional media and social digital media and (3) distrust of technology for tracing contacts and its privacy-related issues. In this short paper, I wish to tackle how these three factors have added up to give rise to a negative public understanding of science in times of a health crisis, such as the current Covid-19 pandemic and finally, how to confront all these problems.
\end{abstract}

Keywords Covid-19 $\cdot$ Science $\cdot$ Misinformation $\cdot$ Peer review system $\cdot$ Digital technology

\section{Introduction}

In this short paper, ${ }^{1}$ I refer to three factors: (1) science's culture of "publish or perish", (2) misinformation spread by traditional media and social digital media and (3) digital technology for tracing contacts in a pandemic and it's privacyrelated issues, which lead to a negative public understanding of science. All these three elements in conjunction lead to a distorted narrative of science, and life science in particular, in times of a health crisis. The discussion in this short

\footnotetext{
1 This short paper belongs to the Topical Collection "Seeing Clearly Through COVID-19: Current and future questions for the history and philosophy of the life sciences", edited by G. Boniolo and L. Onaga.
}

Aníbal Monasterio Astobiza

anibalmastobiza@gmail.com

1 Departamento de Filosofía I, Universidad de Granada, Granada, Spain

2 Visiting Fellow in Global Health and Social Medicine, Center for Bioethics, Harvard Medical School, Harvard University, Boston, USA 
piece centers around science in general, but because it is about how science is working during a time of an emergency or health crisis, it specifically refers to life sciences. In particular, I will explore how the rush to find a vaccine or treatment for the disease can be compromised by the peer review system done quickly and carelessly because of the pressure to find a medical solution. Furthermore, I would like to point out the existence of paradox with digital technology. Digital technology can spread misinformation, but at the same time, this very same technology helps with tracking and monitoring infected people, although some ethical issues of data privacy arise when using digital technology.

Some authors believe that the peer review system that allows the validation of scientific work is in question (Atkinson, 1994; Jukola, 2017) while others see that the reproducibility and replicability of scientific results are almost impossible (Nosek \& Errington, 2020). Still, others think that science communication and outreach to the general public are characterized by hype and sensationalist headlines (Bjørkdahl \& Carlsen, 2017; Dumas-Mallet, 2018). On top of that, the general public no longer sees an expert's status as linked to an authority status (Collins \& Evans, 2007). Finally, trust in public institutions is very low or nonexistent (Stevenson \& Wolfers, 2011). Many of these ailments are not new, but in the wake of the Covid-19 health crisis they have emerged with greater virulence. The InfoSphere (the total space of information), is growing exponentially. The news cycle in the media is dizzying. All these issues do not help science.

During this health crisis, we have seen how science works in real time. However, making sense of scientific work during the pandemic has not been easy, in particular due to the serious problems I am currently discussing. Science is evolving as a living being. Change is natural in science, but this change is very slow. I refer to change in the sense of progress and advancement in knowledge and technology. Confirmation of a scientific hypothesis requires observational and/or experimental studies that take time to prepare correctly. The application of scientific methods requires consensus within the scientific community, and until that consensus is reached, more time is needed. It is a slow process, but one that offers guarantees. This process is distorted when, at a time like the ongoing pandemic, rapid responses are required because the lives of millions of people are at stake (Lipsitch, 2020a, b). The response that science is required to give to society must not only be quick, but also effective. When you want a fast, yet effective solution, but do not solve the problems I have described above is when the problems begin. However, we are fortunate that the process of vaccine development for Covid-19 has been very fast, capitalizing on the enormous existing infrastructure and fostering great collaboration among the scientific community.

The disappearance of the safeguards that normally guarantee the integrity of responsible research and innovation, results from two things happening at once: the academic/scientific culture of "publish or perish" and the need for rapid response in a pandemic context (Grimes, Bauch and Ioannidis, 2018; Smaldino \& McElreath, 2016). According to Marc Lipsitch (2020a, b), these safeguards are:

- Ethics and morals of the researcher. Reporting data and evidence as it is. 
- The peer-review system. The work of editors and researchers who identify failures in methodology or communication of data and results together.

- Transparency. The system of scientific publication must allow free research access to society and other researchers to reproduce, replicate and/or sanction the results.

The work of scientific researchers requires great responsibility due to their activity for society. It requieres responsibility in their activity and profession because it must be guided by the most solid ethical standards and responsibility towards society since research is ususally funded by public money. Under these two approaches, the integrity of research and that of researchers must be defined. A good researcher should not cause harm to his or her research or misinterpret the results of the research. Scientists have to transparently communicate the results of their research and be clear at all stages of the research process. The researcher's main motivation should be to learn and/or discover something, rather than to "prove" something. During the Covid-19 pandemic, we have seen how some of these minimum requirements for responsible research have been overlooked.

The need to obtain rapid solutions to the situation of the Covid-19 health crisis has led to skipping one of the key guarantees for the proper functioning of science: peer-review system. The existence of "pre-print" repositories, in which scientific work has not been formally reviewed by peers, has several advantages. One of them is that it allows to accelerate the scientific process, disseminates ideas, and allows massive access to the results of the research. In short, ideas are accelerated, disseminated, and communicated very quickly thanks to these pre-publication services.

The great disadvantage of these services is that the work achieved by them has insights and findings that must be accepted with great caution (Kwon, 2020). A potential risk is that the media will gain access to them and may influence the opinion and behavior of the public and policymakers alike. Since the beginning of the Covid -19 pandemic, researchers from all over the world, with very good faith and intention, have combined a joint effort to contribute with their knowledge, from social science to biomedicine, to the fight against the new coronavirus. They have published hundreds of research papers that have been poured into these pre-publication repositories. The problem is that science is especially slow, not as an epistemic vice that can be criticized, but because of the need to ensure patient safety and that things work properly.

This has led to duplication of studies (Van Noorden, 2020) which offered flawed arguments (Glasziou Sanders \& Hoffmann, 2020) or, at worst, supported conjectures of potential treatments without any proven effect through randomized clinical trials (Nordling, 2020). Furthermore, this puts the public at risk if all the guarantees of the proper functioning of science are not fulfilled. Science seeks to learn new things about reality. Pursuing this objective sometimes confuses novelty and impact as synonyms of learning. If you look for novelty and impact before learning new things about reality, its possible not to manage data and evidence well, transparency in the process is at stake, and in turn, it is very difficult to replicate results.

The academic culture of "publish or perish" creates a highly competitive environment in securing grants and funding. This encourages a less scrupulous and careful 
modus operandi in research per se, and therefore not very transparent, as the main goal becomes publishing in prestigious journals and obtaining tenure in academic institutions at all costs. The presence of these perverse incentives erodes confidence in science and published research (Oreskes, 2019). Science with a capital "S", the kind of science that discovers and learns from reality and allows lives to be saved, by developing a safe and effective vaccine, cannot fail to be transparent and humble. Otherwise, it can cause harm.

\subsection{Misinformation ("fake news") and digital technology}

In times of a pandemic there is not only a biological virus, there is also a mental virus. The biological virus is the new SARS-CoV-2 coronavirus that causes Covid19 disease. The mental virus is the fear and unfounded alarmist ideas that spread just as fast as the biological virus and leads to infodemics (Jourová, 2020). An infodemic is an avalanche of unconfirmed and often misleading information. The worst part is that among the great amount of information, there are also hoaxes and falsehoods deliberately poured out as a propaganda weapons of misinformation. In recent times, much has been discussed on how to combat misinformation in traditional and digital media (Pennycook \& Rand, 2019). Until recently, researchers have paid very little attention to misinformation in science, much less in the Covid-19 pandemic (Pennycook, 2020). Misinformation or fake news is not only found in the general Infosphere, but also in science-related topics.

Technological solutions have gained importance during the COVID-19 pandemic although digital technology can spread out fake news that travels farther and faster than the truth (Vosoughi et al., 2018) and this also generates infodemics. The implementation of telehealth services and applications for the traceability of infected persons is necessary as a strategic measure to mitigate the pandemic. In addition to promoting the use of telemedicine as a means to prevent community transmission of the virus, the use of human health data for research has been increased. Nonetheless, health data is particularly sensitive data and protected by the European General Data Protection Regulation, and many other legal norms. This has initiated a debate on the ethical and legal limits of digital technology (Gasser et al., 2020). The pandemic has accentuated the dual nature of digital technology that can spread fake news, aswell as erode privacy, and yet help people. Many people question the ability of such trace apps to provide important data on pandemic infections (some think they have many limitations such as a high number of false positives) and see the implementation of digital technology as a new form of surveillance by tech companies and governments (Zuboff, 2019) in the wake of the health crisis caused by the Covid-19 pandemic.

\subsection{The post-Covid-19 world}

It is not easy to solve the problems of science's culture of "publish or perish", misinformation, and the distrust towards technology, but we can implement mitigation strategies and reverse some of these problems that have intensified with the 
Covid-19 health crisis. Regarding science's culture of "publish or perish", we can innovate and suggest bold measures, such as limit the number of articles published per researcher and/or apply other impact requirement measures to asses the quality of science itself (Wang \& Barabasi, 2021). To combat misinformation and the distrust towards technology, we need to make the "truth" more interesting than fake news or conspiracy theories. Science can correct these ailments and misinformation. This will be detected, in part thanks to digital technology that can serve as an instrument with the purpose to help rather than control. In relation to the role of science in major pandemic outbreaks, we could develop a Global Alarm System (and surveillance) to activate large-scale testing (PCR testing or fast diagnosis) and gain a precious amount of time detecting who the people who have been exposed to a virus are and quarantine those who tested positive. With that capacity we can prevent the spread of an epidemic in a country, or a pandemic in the world. This can lead to the advancement in community resilience to pandemics and disasters. We can achieve this through greater scientific cooperation between countries and greater public and private investment in science. Better criteria of what type of scientific research should be prioritized, without ever leaving aside basic research, is also important aswell as re-imagining the communication model and dissemination of science for the general public. With our effort and willingness, better science, better information, and more effective digital technology to face the problems that are threatening humanity as a whole may well be achieved.

Acknowledgements Aníbal Monasterio Astobiza is a co-founder member of $\mathrm{LI}^{2} \mathrm{FE}$ (Laboratorio de Investigación e Intervención Filosófica y Ética). The author gratefully acknowledges the support of the following research projects: EXTEND (H2020 Research Project, ref. 779982), INBOTS (H2020, Research Project, ref. 780073), BIOethAI+ (FI2016-79000-P) and EthAI+3 (PID2019-104943RB-100).

\section{References}

\section{*All links were active at the time of writing}

Atkinson, M. (1994). Regulation of science by “peer review." Studies in History and Philosophy of Science, Part A, 25, 147-158

Bjørkdahl, K., \& Carlsen, B. (2017). Fear of the fear of the flu: Assumptions about media effects in the 2009 pandemic. Science Communication., 39(3), 358-438

Collins, H., \& Evans, R. (2007). Rethinking Expertise. University of Chicago Press.

Dumas-Mallet, E., et al. (2018). Scientific uncertainty in the press: How newspapers describe initial biomedical findings. Science Communication., 40(1), 124-141

Gasser U. et al. (2020). Digital tools against COVID-19: Framing the ethical challenges and how to address them. Arxiv. https://arxiv.org/ftp/arxiv/papers/2004/2004.10236.pdf. Accessed 12 November 2020

Glasziou, P., Sanders, S., \& Hoffmann, T. (2020). Waste in covid-19 research. BMJ, 12(369), 1847. https://doi.org/10.1136/bmj.m1847

Grimes, D., Bauch, C., \& Ioannidis, J. (2018). Modelling science trustworthiness under publish or perish pressure $R$. Soc. open sci., 5, 171511. https://doi.org/10.1098/rsos.171511 
Jourová V. (2020). From pandemic to infodemic. https://ec.europa.eu/commission/presscorner/detail/en/ speech_20_1000. Accessed 20 November

Jukola, S. (2017). A social epistemological inquiry into biases in journal peer review. Perspectives on Science, 25, 124-148

Kwon, D. (2020). How swamped preprint servers are blocking bad coronavirus research. Nature, 581, 130-131. https://doi.org/10.1038/d41586-020-01394-6

Lipsitch M. (2020). Good science is good science. Boston Review. Retrieved from [http://bostonreview. net/science-nature/marc-lipsitch-good-science-good-science]

Lipsitch M. (2020). [ June, 6th, 2020] In light of the retractions it's worth remembering: Peer review is one imperfect part of the at-least 4-part safety net that keeps science functioning. Layer 1 is basic ethics among investigators: don't make up or misrepresent data [Tweet]. Retrieved from https://twitt er.com/mlipsitch/status/1269260948369440779

Nordling, L. (2020). Unproven herbal remedy against COVID-19 could fuel drug-resistant malaria, scientists warn. Science. https://doi.org/10.1126/science.abc6665

Nosek, B., \& Errington, T. (2020). The best time to argue about what a replication means? Before you do it. Nature, 583, 518-520

Oreskes, N. (2019). Why trust science? Princeton University Press.

Pennycook, G., et al. (2020). Fighting COVID-19 Misinformation on Social Media: Experimental Evidence for a Scalable Accuracy-Nudge Intervention. Psychological Science, 31(7), 770-780

Pennycook, G., \& David, G. R. (2019). Fighting misinformation on social media using crowdsourced judgments of news source quality. PNAS, 116(7), 2521-2526

Smaldino, P., \& McElreath, R. (2016). The natural selection of bad science. $R$ Soc Open Sci. https://doi. org/10.1098/rsos. 160384

Stevenson, B., \& Wolfers, J. (2011). Trust in public institutions over the business cycle. American Economic Rewiew., 101(3), 281-287

The age of surveillance capitalism: The fight for a human future at the new frontier of power. Profile Books, London.

Van Noorden, R. (2020). Pioneering duplication detector trawls thousands of coronavirus preprints. Nature. https://doi.org/10.1038/d41586-020-02161-3

Vosoughi, S., Roy, D., \& Aral, S. (2018). The spread of true and false news online. Science, 359(6380), 1146-1151. https://doi.org/10.1126/science.aap9559

Wang, D., \& Barabasi, A. (2021). The science of science. Cambridge University Press.

Publisher's Note Springer Nature remains neutral with regard to jurisdictional claims in published maps and institutional affiliations. 